\title{
Discriminant analysis of selected edible fats and oils and those in biscuit formulation using FTIR spectroscopy.
}

\begin{abstract}
Recently, lard adulteration has highlighted the importance of checking raw materials and food products in order to assure their authenticity. Discriminant analysis (DA) using selected frequency regions $(3,050-2,800,1,800-1,600$, and 1,500-650 cm-1) was exploited for the classification of lard and other commercial vegetable oils and animal fats. As a result, the Cooman plot showed that all vegetable fats/oils and animal fats, including lard, are clustered in a distinct group. DA was also employed to assign real food samples, in particular biscuit, into one of two groups. In addition, DA is a very useful means for Halal screening technique in order to enhance the Halal authentication process.
\end{abstract}

Keyword: Discriminant analysis; Fats and oils; FTIR spectroscopy; Halal authentication; Lard. 